\title{
IMPROVING PLASMA SHAPING ACCURACY THROUGH CONSOLIDATION OF CONTROL MODEL MAINTENANCE, DIAGNOSTIC CALIBRATION, AND HARDWARE CHANGE CONTROL
}

\author{
by \\ D.S. BAGGEST, D.A. ROTHWEIL, S. PANG, \\ M.L. WALKER, and A. NEREM
}

SECEIVED

APS 031997

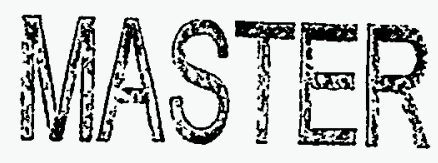

OSTI

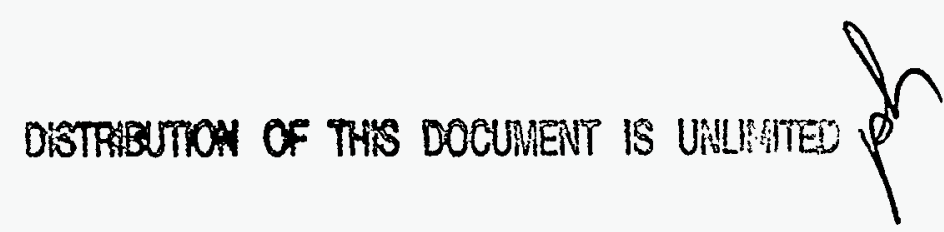

DECEMBER 1995 


\section{DISCLAIMER}

This report was prepared as an account of work sponsored by an agency of the United States Government. Neither the United States Government nor any agency thereof, nor any of their employees, makes any warranty, express or implied, or assumes any legal liability or responsibility for the accuracy, completeness, or usefulness of any information, apparatus, product, or process disclosed, or represents that its use would not infringe privately owned rights. Reference herein to any specific commercial product, process, or service by trade name, trademark, manufacturer, or otherwise, does not necessarily constitute or imply its endorsement, recommendation, or favoring by the United States Government or any agency thereof. The views and opinions of authors expressed herein do not necessarily state or reflect those of the United States Government or any agency thereof. 


\section{DISCLAIMER}

Portions of this document may be illegible in electronic image products. Images are produced from the best available original document. 


\title{
IMPROVING PLASMA SHAPING ACCURACY THROUGH CONSOLIDATION OF CONTROL MODEL MAINTENANCE, DIAGNOSTIC CALIBRATION, AND HARDWARE CHANGE CONTROL
}

\author{
by \\ D.S. BAGGEST, D.A. ROTHWEIL, S. PANG, \\ M.L. WALKER, and A. NEREM
}

This is a preprint of a paper presented at the 16th IEEE/NPSS Symposium on Fusion Engineering, September 30-October 5, 1995, Champaign, Illinois, and to be printed in the Proceedings.

\author{
Work supported by \\ U.S. Department of Energy \\ Contract DE-AC03-89ER51114
}

\section{GENERAL ATOMICS PROJECT 3466 \\ DECEMBER 1995}




\title{
Improving Plasma Shaping Accuracy Through Consolidation of Control Model Maintenance, Diagnostic Calibration, and Hardware Change Control*
}

\author{
D.S. Baggest, D.A. Rothweil, S. Pang, M.L. Walker, A. Nerem \\ General Atomics \\ P.O. Box 85608, San Diego, California 92186-9784
}

\section{ABSTRACT}

With the advent of more sophisticated techniques for control of tokamak plasmas comes the requirement for increasingly more accurate models of plasma processes and tokamak systems. Development of accurate models for DIII-D power systems, vessel, and poloidal coils is already complete, while work continues in development of general plasma response modeling techniques. Increased accuracy in estimates of parameters to be controlled is also required. It is important to ensure that errors in supporting systems such as diagnostic and command circuits do not limit the accuracy of plasma parameter estimates or inhibit the ability to derive accurate plasma/tokamak system models. To address this issue, we have developed more formal power systems change control and power system/magnetic diagnostics calibration procedures. This paper discusses our approach to consolidating the tasks in these closely related areas. This includes, for example, defining criteria for when diagnostics should be re-calibrated along with required calibration tolerances, and implementing methods for tracking power systems hardware modifications and the resultant changes to control models.

\section{INTRODUCTION}

Advanced plasma control algorithms are presently under development at DIII-D. A model based multiple-input-multiple-output (MIMO) controller utilizing feedback in the form of coil currents, power supply voltages, magnetic flux, and fields will be implemented into the present plasma control system (PCS) [1]. Controller performance depends on the accuracy of system models developed from test validation data. Inaccuracies in diagnostic systems can produce errors in both model validation data and acquired data used by the PCS during plasma operations. At DIII-D, calibration responsibilities for many diagnostics presently belong to the "owners" of the equipment. An integrated calibration system for DIII-D hardware used by the PCS is therefore being developed. This system addresses the issues of calibration system organization, accuracy requirements of calibration tests, test procedures, test equipment, and model change control. The PCS should be viewed as an essential DIII-D support system with the calibration support structure to maintain its optimum performance. In this paper, we describe the organization of the plasma control calibration maintenance system (PCCMS), calibration test procedures implemented and proposed, calibration results, and tools developed for maintaining the PCS.

\section{THE DIII-D INTEGRATED CALIBRATION SYSTEM}

The function of the PCCMS is to link diagnostic calibrations with controller model maintenance. Shown in Fig. 1 is a block diagram of the Plasma Control Calibration Maintenance System structure. The PCCMS is divided into two major sections, hardware calibration and control model maintenance. Hardware calibration includes both DIII-D power systems and magnetic diagnostics utilized by the PCS. Documented calibration results can be linked through the calibration database to controller model maintenance. In the power systems area we include the power supplies utilized by the PCS for plasma control. They are identified as the E-supply (ohmic heating), the F-supplies (field shaping), and the dc switching regulators (choppers). Buss voltage monitors are also grouped within the power systems area. Included in the magnetic diagnostics area are the various diagnostics utilized

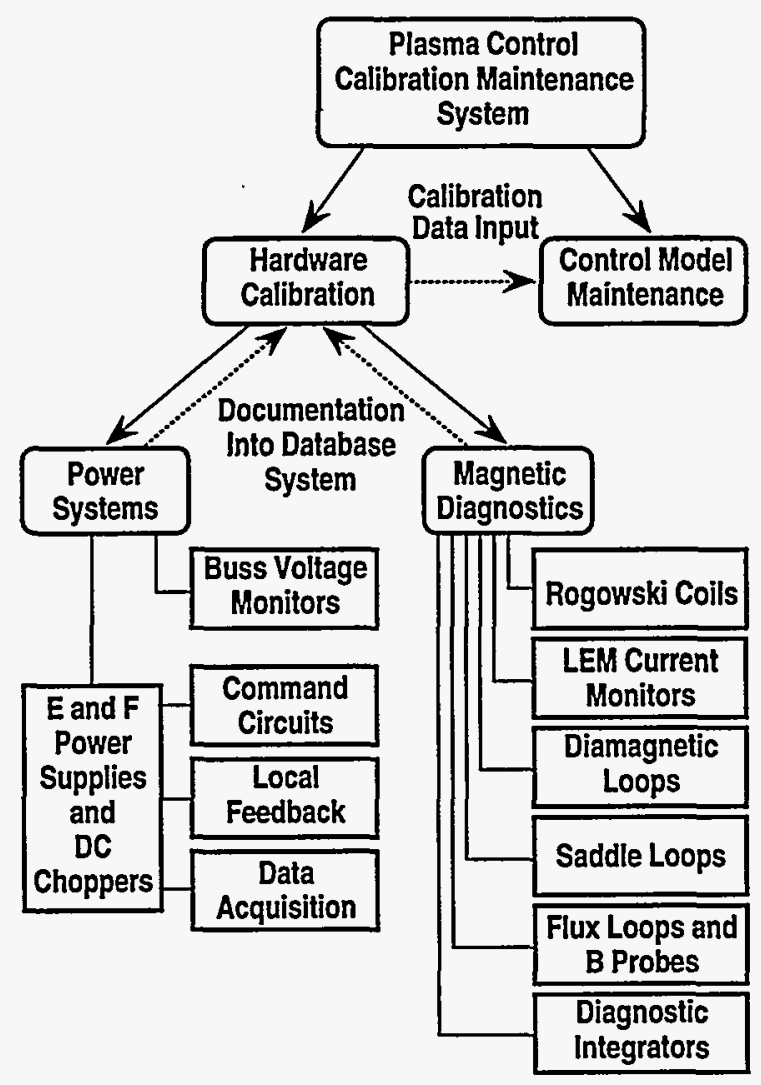

Fig. 1. PCCMS structure.

\footnotetext{
*Work supported by the U.S. Department of Energy under Contract No. DE-AC03-89ER51114.
} 
by DIII-D for measuring fields and currents. The power and magnetic diagnostics are discussed in the following sections.

The inclusion and grouping of DIII-D hardware into the PCCMS establishes a method of single point coordination for equipment calibrations affecting plasma control. Persons responsible for the diagnostic hardware design and installation along with designers of the control system specify the calibration accuracy required for each diagnostic. In Table I, we present the accuracy requirements determined for the separate diagnostics.

Table I

Accuracy Requirements for Diagnostics

\begin{tabular}{lc}
\hline \multicolumn{1}{c}{ Diagnostic } & Required Accuracy \\
\hline Rogowski Coils & $0.5 \%$ \\
LEM current monitors (C-coil) & $0.5 \%$ \\
LEM current monitors (choppers) & $10.0 \%$ \\
Diamagnetic loop & $<1 \%$ \\
Flux loops \& B-probes & $0.5 \%$ \\
Saddle loops & $10 \%$ \\
Calibvolt divider network & $0.1 \%$ \\
Command signals & $1 \%$ \\
Voltage \& current feedback & $1 \%$ \\
Buss voltage monitors & $2 \%$ \\
\hline
\end{tabular}

\section{A. Magnetic Diagnostics}

Rogowski coils are used to measure currents in the E-, F-, and B-coils. In addition, three Rogowski coils are used to measure total current in the tokamak vessel and plasma. Currents in the C-coils are measured with six LEM current monitors. The diamagnetic loops are three separate single loop wires routed through the center and outside of the tokamak vessel in the vertical plane. These loops measure the total toroidal flux in the tokamak. The saddle loops are used to measure local flux through the vessel. flux loops and B-Probes are used to measure poloidal flux and flux density. All magnetic diagnostic voltage signals, except for LEM current monitors, are processed through Volt-second integrators to convert voltage signals to "physical" units.

\section{B. Power Systems}

The calibration of power systems hardware is divided into three separate areas of operation: command signal accuracy, local feedback, and data acquisition. Fig. 2 shows a simplified diagram of the signal paths for a typical F-supply and dc chopper.

The power supply command signals originating from the PCS are routed through isolation amplifiers to the power supply regulator boards (Fig. 2, label 1). The chopper command signals are sent to chopper control modules (18 total). The command signals are acquired and digitized by the DIII-D acquisition system (Fig. 2, label 2). Voltage and current feedback signals in the F- and E-supplies are utilized locally by the power supply regulator boards for control Fig. 2, label 3). On the F-supplies, both the voltage and current feedback signals are acquired from the regulator

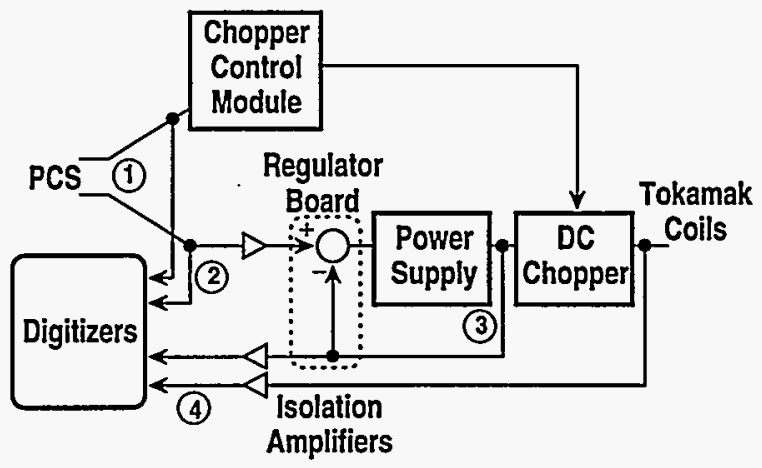

Fig. 2. F-supply and dc chopper signal paths.

board for digitization into the data acquisition system (Fig. 2, label 4). The E-power supply utilizes a separate circuit for output voltage data acquisition. Voltage and current signals for both the E- and F-supplies are passed through isolation amplifiers before digitization.

The output voltages and currents of the dc choppers are not used for local control but are acquired through isolation amplifiers and digitized by both the data acquisition digitizers and separate plasma control digitizers utilized by the PCS. In addition to the chopper output voltage and current monitoring, there are chopper over-current protection circuits. These systems use shunts on the chopper outputs and turn the choppers off in the event of an over-current situation. In addition, voltage monitors have recently been added to measure the three busses joining the E- and F-coils.

\section{DEVELOPMENT OF CALIBRATION PROCEDURES}

In this section, we discuss the development of test procedures for calibrating the diagnostics included in the PCCMS. All of these diagnostics have been calibrated some time in the past, but not as part of a regularly scheduled maintenance procedure. Several systems had procedures, but were not regularly calibrated due to difficulty or costs in both personnel time and test equipment. To decrease costs and increase accuracy, we have developed several new calibration procedures while streamlining some older ones. The results are calibrations done as preventive rather than corrective maintenance.

\section{A. Rogowski Coils}

Rogowski coils measure currents from $10 \mathrm{kA}$ to $200 \mathrm{kA}$ in the tokamak coils and provide voltage measurement signals for integration within a $\pm 10 \mathrm{~V}$ range. The Rogowski coils on DIII-D were last calibrated approximately eight years ago prior to installation. Testing and calibration at that time involved using a high current amplifier to drive a sine wave signal through a wire looped through the Rogowski fifty times. The output voltage of the Rogowski was monitored as was the current and frequency of the signal applied to the 50 turn test loop. Data was acquired at several different frequencies to establish linearity with frequency. Each Rogowski coil has a constant $(\mathrm{K})$ associated with it which is determined by 
its construction. The value of $\mathrm{K}$ is used by data acquisition software to convert the voltage output signal of the Rogowski coil to a "measured" current value. To solve for the constant $(\mathrm{K})$, the following calculations are done [2].

$$
\mathrm{V}_{\text {rog }}=\mathrm{It} * \omega * \mathrm{~K}
$$

where $V_{\text {rog }}=$ Rogowski output voltage $(V)$

$$
\begin{aligned}
& \mathrm{It}=\text { ac current in Rogowski test loop }(\mathrm{A}) \\
& \omega=\text { Frequency of test loop signal }(1 / \mathrm{s}) \\
& \mathrm{K}=\text { Rogowski constant (V-s/A) }
\end{aligned}
$$

As with all calibration tests, measurement accuracy is determined by that of the test equipment. For our re-calibration of the Rogowskis, all equipment used was verified as calibrated and having an accuracy within $0.3 \%$. The procedure used for the initial calibration eight years ago was modified slightly for our test. Shown in Fig. 3 is a diagram of the test set-up.

A portable test loop was constructed with a 50 pin " $D$ " connector with 18 awg wire looped around itself 50 times. The test loop was connected back on itself through the Rogowski coil. Current in the test loop was monitored with a calibrated current transformer. The frequency of the test signal and output voltage of the Rogowski were also recorded for analysis.

Original Rogowski calibrations were done as a bench test, while our tests were conducted with the Rogowski coils installed on their respective buss-work (E- and B-coils) or cables (F-coils). Recent modifications at DIII-D required the installation of several new Rogowski coils on E-coil busswork. This provided an opportunity to compare a Rogowski constant determined from bench testing with one obtained after installation on buss-work. A deviation of almost 1\% between the two constant values was discovered, exceeding the Rogowski accuracy limit of $0.5 \%$. Measurement tests done for Rogowski coils installed on F-coil cables displayed an accuracy within $0.3 \%$ of the original calibration values for all but two of the coils. All Rogowski coils tested had standard deviations less than $0.3 \%$ over the frequency range

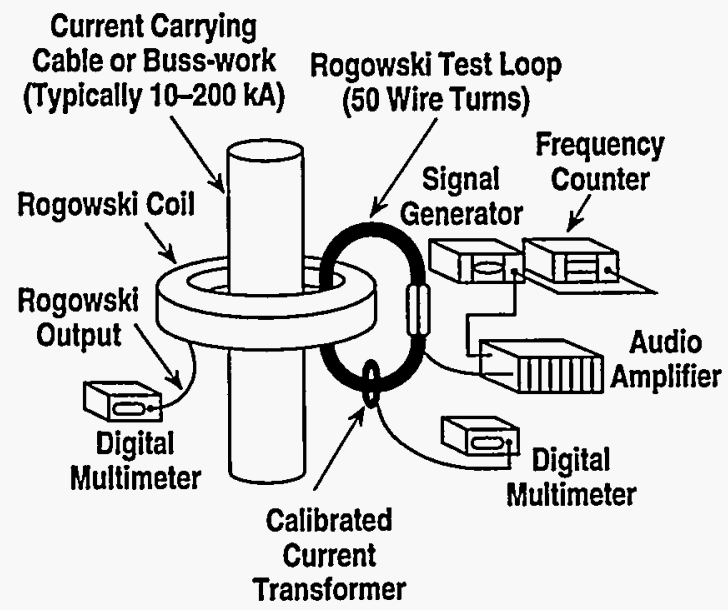

Fig. 3. Example of Rogowski test setup. of 500 to $2000 \mathrm{~Hz}$. A second calibration of buss-work installed Rogowskis will be done to verify results. Results will determine if future Rogowski calibrations will require removal from buss-work.

\section{B. LEM Current Monitors}

LEM current monitors are used on the $\mathrm{C}$-coils and the chopper outputs (over-current interlocks). A new calibration procedure is being developed from a test method used to calibrate hall effect current probes. Current transformers are used to induce a current (up to $3 \mathrm{kA}$ ) into a test loop containing the LEM current monitors. A calibrated shunt is used to monitor the current in the test loop and provide a calibration standard. This test procedure is still under development, therefore the C-coil and chopper LEM's have not yet been calibrated.

\section{Diamagnetic, Saddle, Flux Loops and B-Probes}

The diamagnetic and saddle loops were last calibrated in March 1994 [3]. Calibration for this system consisted of energizing the $F, E, B, C$, and the $n=1$ coils individually to determine the flux picked up by the diamagnetic loops. At least 28 total test "shots" and two days of tokamak operations were required to individually test for linear and non-linear field pickups from each coil. Calibration of the flux loops and B-probes was accomplished during control model development. Individual coil response tests provided data for calibrating Green functions used to map coil currents to fields and coil mutual inductance to flux loops.

\section{Integrators and Calibvolt}

The integrators are calibrated daily before operations for offset, gain, drift, and noise. A square wave signal is generated and acquired by software as the point name CALIBVOLT. This signal's accuracy is verified in software and applied to each integrator through a voltage divider network. Because there are several different gain values associated with the integrators, the divider converts the calibvolt signal to multiple voltage levels read by the integrators. The integrator outputs are monitored and compared with the expected outputs as determined by software. Any integrator out of calibration is automatically flagged for adjustment. The accuracy of the voltage divider which delivers the correct voltage to the integrators has recently been tested. This .was accomplished by applying the calibvolt signal to the resistive divider and monitoring the divider output values with a storage oscilloscope. The ratio values in software were subsequently adjusted to reflect the actual divider ratios.

\section{E. Power Supply Command, Feedback, and Data Acquisition Signals}

Calibration procedures for the power supply and chopper systems has in the past been a laborious process requiring a high allocation of technician time and test equipment. Systems were checked individually while measurements were made and recorded by hand, increasing the possibility of error. New procedures are being developed to address these issues. 
The viability of a PC based system coupled with a high voltage amplifier and digital storage oscilloscopes is being researched. The PC would function as both a control and data recording tool. Several systems could be calibrated simultaneously increasing the test efficiency. The elimination of manual data recording would increase calibration accuracy.

\section{F. Buss Voltage Monitors}

The buss voltage monitors were recently installed and calibrated. Scheduling for follow-up calibration has yet to be determined. The functionality of the PC based system (see Section E) could be applied to the calibration of these voltage probes.

\section{CALmRATION AND MODEL MAINTENANCE}

Development of a software based calibration update system is underway. A final step included in the calibration of the hardware systems described will be the entry of calibration measurement data into the PCCMS calibration database. This will provide a centralized reference point for tracking equipment status beyond simply updated calibration values. Examples include, last known calibration dates, personnel involved, modifications to equipment, and calibration schedules. With easy access by technicians, data entry after new calibration adjustments will become a routine part of hardware calibrations. After review of adjustments and calibrations, plasma control models will be updated to represent new calibration values. To also ensure hardware upgrades are done in conjunction with model maintenance, a review of system modifications and their impact on plasma control functionality will be included in future design reviews. The models can then be changed and re-calibrated to reflect the modification. This will maintain the plasma control system model accuracy and eliminate the effects of unknown modifications.

In addition to a software database, we are developing a program to compare daily system performance to that predicted by system models. This facilitates a regular check of model accuracy and automated detection of malfunctioning equipment. The comparison program also provides a fast but less accurate check of diagnostic calibration. In Fig. 4 we show two sample plots of the $D_{2}$ power supply as generated by the comparison program. Actual power supply output voltage data is plotted on top of simulated output data generated by power supply models. Command signals for the power supplies are used as input to the models. In these example plots, the power supplies were turned off by hardware interlocks after approximately $2.3 \mathrm{~s}$. Power supply command signals continued to be generated, as indicated by the model output response plots. Acquired command signal data can also be overlaid on the plots for futher verification or analysis.

An example of the programs use as an error detection tool is shown in the second plot (shot 87110). The supply is sent a command signal, verified by the model output response or an overlay of the command signal, and fails to respond as predicted by the model output. There also seems to be a delay in the acquired data during the initial turn on. This is being investiated.
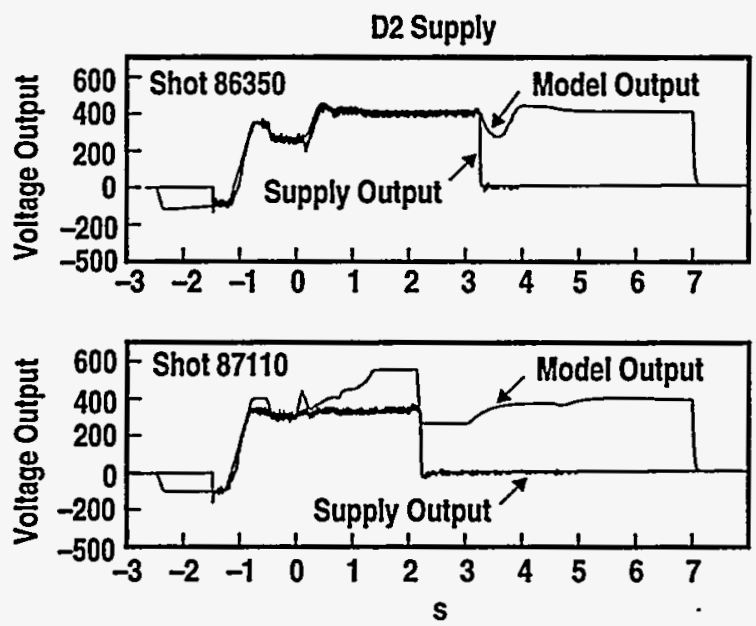

Fig. 4. Example output of model comparison program for F-power supply $\mathrm{D}_{2}$.

\section{CONCLUSIONS}

A Plasma Control Calibration Maintenance System (PCCMS) is under development in parallel with advances in plasma control. Regular equipment calibrations are a necessity for optimum performance in any system. The regular calibration of DIII-D diagnostic hardware will improve accuracy not only in plasma control but also in the collected data used for plasma analysis. A software database will maintain a centralized documentation system enabling changes to be tracked while providing a link for control model maintenance. The goal of the PCCMS is to maintain PCS performance through preventive rather than corrective methods

\section{REFERENCES}

[1] M.L. Walker, "Toward integrated digital control of the DIII-D Tokamak," in Proc. 15th IEEE/NPSS Symp. on Fusion Engineering, pp. 119-122, 1993.

[2] E.J. Strait, private communication.

[3] R.J. LaHaye, General Atomics Mini-Proposal No. D3DMP 304, February 1994. 